\title{
LETTER
}

\section{Long non-coding RNA tagging and expression manipulation via CRISPR/Cas9-mediated targeted insertion}

\section{Dear Editor,}

Long non-coding RNAs (IncRNAs), defined as RNA transcripts longer than 200 nucleotides without the protein-coding ability (Carninci et al., 2005), share many features with protein-coding messenger RNAs (mRNAs) such as polyadenylated 5 ' ends and multi-exonic structures (Guttman et al., 2010). Though expression levels are less abundant, IncRNAs outnumber mRNAs with more diverse regulatory functions (Quinn and Chang, 2016). They may serve as decoys, sponges, signals or scaffolds in regulating chromatin conformation, nuclear organization, gene expression, and protein activity in cis or trans manner (Ulitsky and Bartel, 2013; Quinn and Chang, 2016). LncRNAs are involved in various physiological processes and their loss- or gain-offunction mutations have been implicated in the pathogenesis of human diseases (Wapinski and Chang, 2011). Although their functions have been investigated extensively, manipulation of IncRNAs is challenging, limiting further in-depth analysis for IncRNAs. Efficient and convenient tagging method could be helpful for effective IncRNAs immunoprecipitation to explore IncRNAs-DNA/RNA/protein interactions (Engreitz et al., 2014; Chu et al., 2015). Another challenge is IncRNAs expression manipulation with high efficiency and specificity: Point mutations or insertions and deletions (Indels) are usually insufficient to block IncRNAs functions completely (Cong et al., 2013; Mali et al., 2013). Deleting the whole IncRNA loci or changing IncRNA expression with either clustered regularly interspaced short palindromic repeats (CRISPR)-associated endonuclease Cas9 system, CRISPR interference (CRISPRi) or CRISPR activation (CRISPRa) system have been developed as alternative approaches (Zhu et al., 2016; Liu et al., 2017). However, many IncRNA loci overlap with protein-coding genes and even share common promoter regions, restricting the applications of available tools. In addition, many IncRNAs are cisacting factors, so traditional overexpression strategy may not work in such conditions. Recently it is shown that targeted insertion could be achieved with CRISPR/Cas9 system via canonical non-homologous end joining (c-NHEJ) pathway without the need for homologous or microhomologous sequences (Schmid-Burgk et al., 2016; Suzuki et al., 2016), so it is plausible to achieve targeted insertion at different sites with one universal donor vector using CRISPR/Cas9 system. As gene trap system has been well-established to disrupt gene functions with selection markers/tags for subsequent functional analysis (Stanford et al., 2001), we here modified gene trap vectors and used CRISPR/Cas9 to establish a scalable tool entitled CTRL (CRISPR-mediated tagging and regulation of IncRNAs) for IncRNA tagging and expression manipulation in mammalian cells. With this method, we successfully tagged IncRNAs at either $5^{\prime}$ or $3^{\prime}$ end. And IncRNA expression status was either stimulated or inhibited reversibly depending on the targeted insertion site.

CTRL system contains a modified gene trap vector, a plasmid expressing S. pyogenes Cas9 (SpCas9) and two sgRNAs driven by two U6 promoters respectively (one genome-targeting sgRNA and another donor plasmid-targeting sgRNA) (defined as Cas9-2sgRNA) for IncRNA tagging and expression manipulation purposes. In principle, modified gene trap vector and Cas9-2sgRNA were transfected simultaneously into 293T cells for donor DNA plasmid linearization and targeted insertion at desired genome locus (Fig. 1A). For targeted insertion near transcriptional termination site, a modified polyA trap vector containing CMVpuromycin selection cassette without polyA signal, a specific sgRNA targeting site and $4 \times M S 2$ or $24 \times$ MS2 tagging sequences were designed (Fig. S1A and S1B). With targeted insertion near transcriptional termination site, puromycin expression is induced to serve as selection marker for cells containing established targeted insertion. Initially, to determine the applicability of CTRL system for IncRNA tagging and purification, genome-targeting sgRNA inside transcriptional termination site of phosphatase and tensin homolog pseudogene 1 (PTENP1) was designed. Then modified polyA trap vector containing $24 \times \mathrm{MS} 2$ tags and Cas9-2sgRNA/PTENP1 were transfected into 293T cells. After $48 \mathrm{~h}$, puromycin was added at a final concentration of $2 \mu \mathrm{g} / \mathrm{mL}$ and cells were cultured for another 4 days. Survival cells were further incubated in normal growth medium without puromycin for about 1 week to obtain sufficient cells for subsequent analysis. Established targeted insertion and 
A

Linearized targeting vector

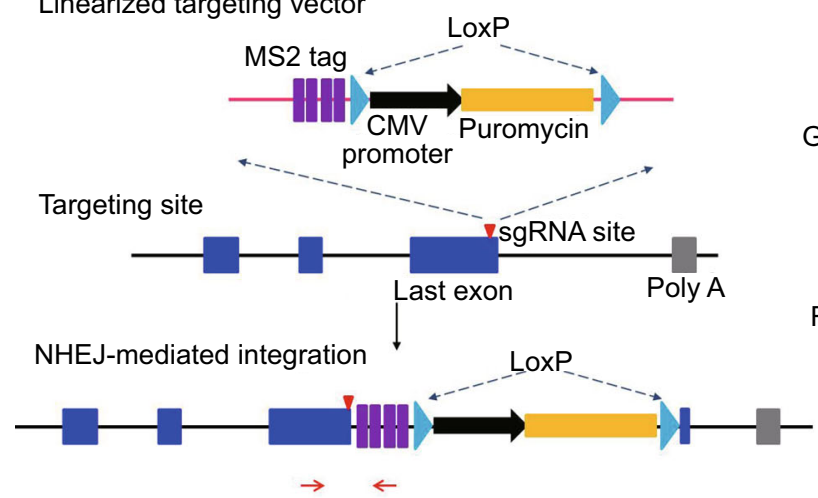

D

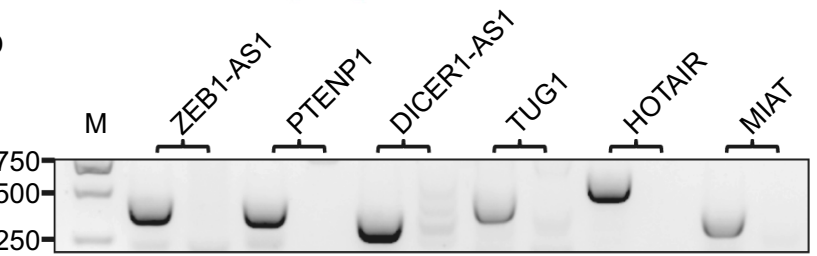

500

250

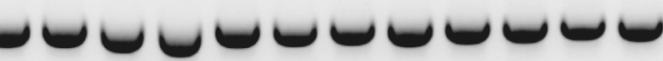

K.I. N.C. K.I. N.C. K.I. N.C. K.I. N.C. K.I. N.C. K.I. N.C.
$\mathrm{F}$

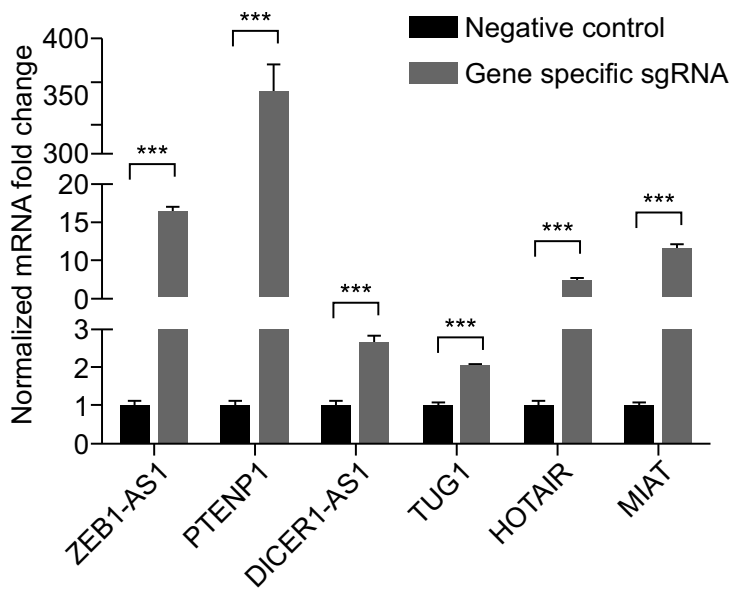

B

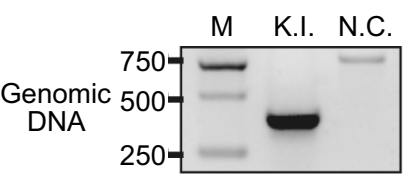

C

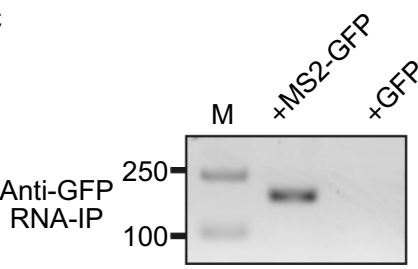

RNA

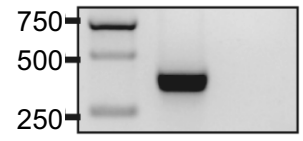

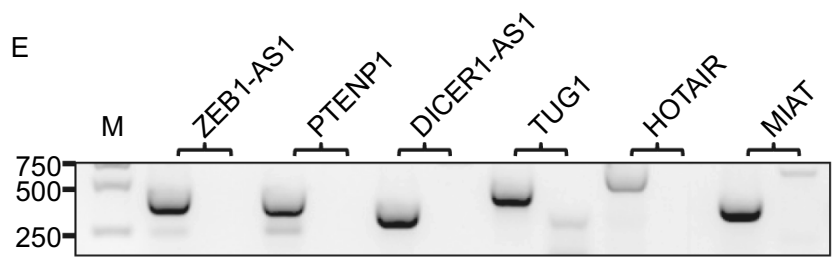

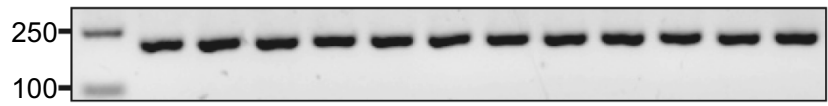

K.I. N.C. K.I. N.C. K.I. N.C.K.I. N.C.K.I. N.C. K.I. N.C.

G

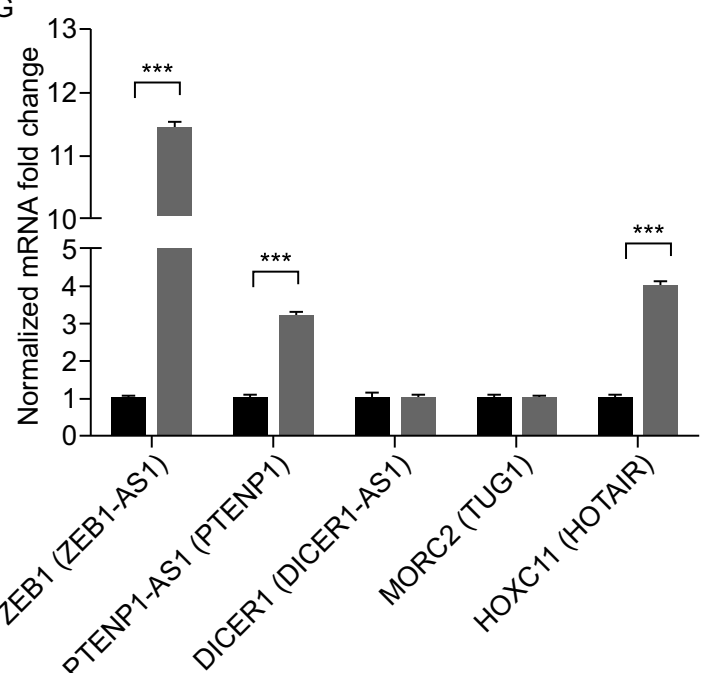

IncRNA tagging were confirmed at the genomic level and mRNA level with PCR/RT-PCR, respectively (Fig. 1B). For PTENP1 purification, 293T cells with established targeted insertion were transfected with plasmid expressing either EGFP or MS2-EGFP fusion protein and cultured for 4 days. Then RNA-immunoprecipitation (RNA-IP) was performed using GFP-Trap_A beads. We found that PTENP1 transcripts were significantly enriched in cells expressing MS2EGFP fusion protein as compared with cells expressing EGFP protein (Fig. 1C), confirming the reliability of CTRL system for IncRNA purification. Whether CTRL system could be used to manipulate IncRNA expression was further investigated with designed sgRNAs inside transcriptional termination of six IncRNAs ZEB1 antisense RNA1 (ZEB1AS1), PTENP1, DICER1 antisense RNA 1 (DICER1-AS1), taurine up-regulated 1 (TUG1), HOX transcript antisense RNA (HOTAIR), and myocardial infarction associated transcript (MIAT). As a small amount of puromycin proteins may be produced from CMV-puromycin selection cassette without polyA signal in cells containing targeted insertion with 
Figure 1. LncRNA tagging and stimulation by CRISPR/ Cas9-mediated targeted insertion inside transcriptional termination. (A) A scheme for CRISPR/Cas9-mediated targeted insertion inside IncRNA transcriptional termination. Circular modified gene trap vector is initially linearized by vectortargeting Cas9-sgRNA complex. Simultaneously double strand breaks (DSBs) are induced inside transcriptional termination site by genome-targeting Cas9-sgRNA complex. Then linearized vector could be integrated into targeting site via NHEJ mechanism. Red arrow pair shown here represents primers used for PCR confirmation of targeted insertion. (B) DNA agarose gel analysis of targeted insertion for PTENP1 tagging with 24× MS2 tags at both DNA and RNA level with PCR and RT-PCR respectively in 293T cells treated as (A) and cultured for 1-2 weeks after puromycin selection. (C) DNA agarose gel analysis of PTENP1 mRNA purification in 293T cells with RTPCR. PTENP1 transcripts were purified with GFP-Trap_A beads in 293T cells with established targeted insertion transfected with either MS2-EGFP or EGFP expression vector for 4 days. (D) PCR analysis of targeted integration for six different IncRNAs using genomic DNA of 293T cells with established targeted insertion. (E) RT-PCR analysis of IncRNA tagging with $4 \times$ MS2 tags using total RNA of 293T cells with established targeted insertion. (F) Quantitative PCR analysis of targeted IncRNA expression in 293T cells with established targeted insertion. (G) Quantitative PCR analysis of neighboring genes' expression in 293T cells with established targeted insertion. In $(F)$ and (G), 293T cells without treatment were used as negative control. GAPDH was used as internal control. All experiments were performed independently three times and one representative result was shown. ${ }^{* * *} P<0.005$ (two-sided Student's $t$-test).

reverse direction, we picked out 2-5 clones for each IncRNA and examined the ratio of clones containing insertion with correct direction. We found that the ratios of targeted insertion with right direction were at least $50 \%$, with 3/6 IncRNAs showing $100 \%$ targeted insertion with right direction (Table S1). Established targeted insertion with right direction was confirmed at the genomic level with PCR (Fig. 1D). Then Sanger sequencing was used to examine detailed sequences surrounding the targeted sites for each IncRNA and revealed that targeted insertion was quite precise, as fusion sites in 11/12 clones are generated as expected (Fig. S2). LncRNA tagging was confirmed at mRNA level with RT-PCR (Fig. 1E). The expression level of targeted IncRNAs in 293T cells was examined using real-time quantitative PCR. We found that expression of all six IncRNAs was upregulated significantly, with at least 2-fold changes in 293T cells with established targeted insertion (Fig. 1F). The observed IncRNA upregulation is consistent with previous work reporting that polyadenylation promoted IncRNA turnover (Beaulieu et al., 2012). Many IncRNAs are involved in gene expression regulation with neighboring genes as targets, so the expression of corresponding neighboring genes
(ZEB1 vs. ZEB1-AS1; PTENP1-AS1 vs. PTENP1, DICER1 vs. DICER1-AS1, MORC2 vs. TUG1, HOXC11 vs. HOTAIR) was examined. It was revealed that the expression of ZEB1, PTENP1-AS1, and HOXC11 was induced significantly, while DICER1 and MORC2 changed little in 293T cells with established target insertion (Fig. 1G), which indicated that IncRNAs induced by CRISPR/Cas9-mediated targeted insertion were functional.

Targeted insertion inside transcriptional termination site is suitable for IncRNA tagging. However, exogenous fragment insertion inside IncRNA transcripts might disrupt IncRNA functions. Then we designed another six sgRNAs targeting sites after IncRNA transcriptional termination (ZEB1-AS1, PTENP1, DICER1-AS1, TUG1, HOTAIR, and MIAT) and examined the impact of targeted insertions on IncRNA expression in 293T cells (Fig. S3A). Established targeted insertions were confirmed at the genomic level with PCR for all six IncRNAs as described above (Fig. S3B). The expression of all six targeted IncRNAs was also upregulated as described above, though increasing extent was not so dramatic as comparing to cells with targeted insertion inside transcriptional termination site of related IncRNAs (Fig. S3C). The expression changes of neighboring genes were similar as described above, though the upregulation of ZEB1, PTENP1-AS1, and HOXC11 was not so robust as comparing to cells with targeted insertion inside transcriptional termination site of related IncRNAs (Fig. S3D).

Reversion of the established mutation, also referred as rescue experiments, is critical for functional confirmation of targeted genes. Here we added two LoxP sites in the same direction at two ends of linearized donor DNA, so established targeted insertion could be depleted via Cre recombinase delivery (Fig. 2A and 2B). 293T cells with established targeted insertion near transcriptional termination sites of IncRNAs were transfected with Cre expression vector and cultured for about 4 days. Expression changes of targeted IncRNAs and neighboring genes were examined. We found that Cre expression reversed the upregulation of IncRNAs and IncRNA-regulated gene targets in 293T cells with established targeted insertion inside or after transcriptional termination site partially (Fig. 2C-F).

As targeted insertion near transcriptional termination sites stimulated IncRNA expression, we wondered whether targeted insertion at transcriptional start site would also change IncRNA expression. Here a modified promoter trap vector containing puromycin-polyA cassette without promoter, a specific sgRNA targeting site and 4× MS2 tagging sequences were designed for targeted insertion at transcriptional start site (Fig. S1C). Puromycin-polyA cassette without promoter would be stimulated by endogenous IncRNA promoter in cells with established targeted insertion, which could be depleted by Cre-mediated recombination (Fig. S4A). Six sgRNAs targeting IncRNA transcriptional start sites (ZEB1AS1, PTENP1, DICER1-AS1, TUG1, HOTAIR, and MIAT) were designed and their impact on IncRNA expression was 
A Linearized targeting vector

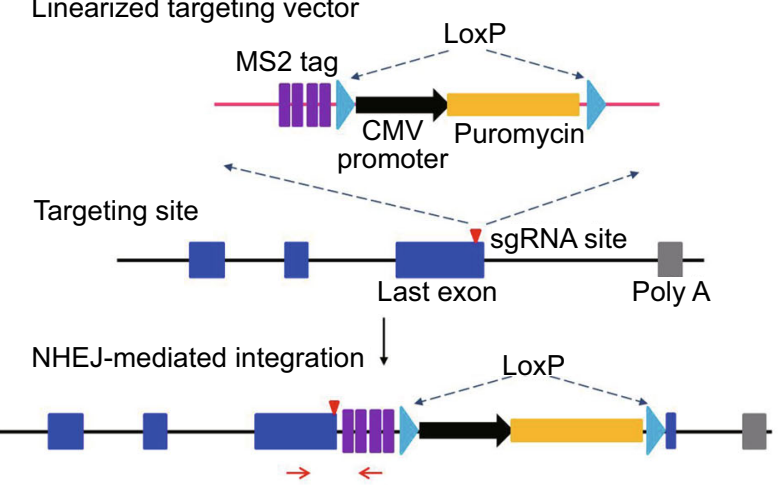

Cre-mediated recombination

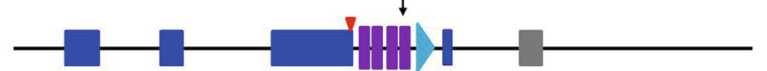

C

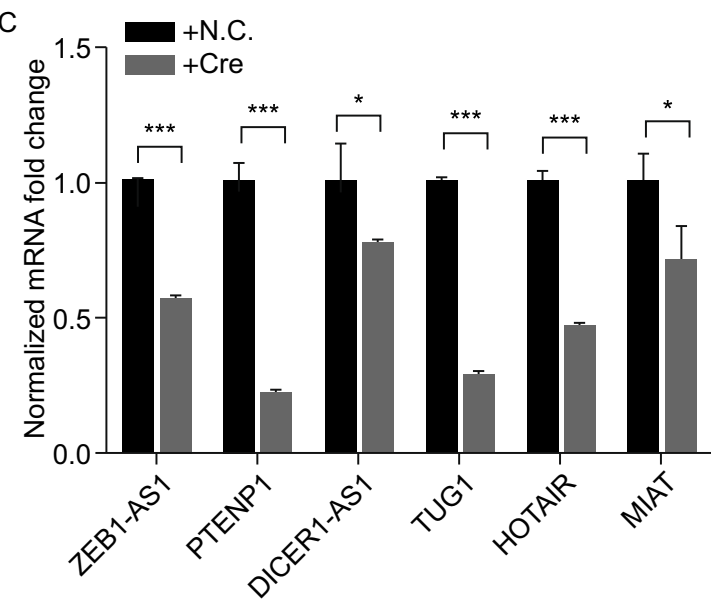

E

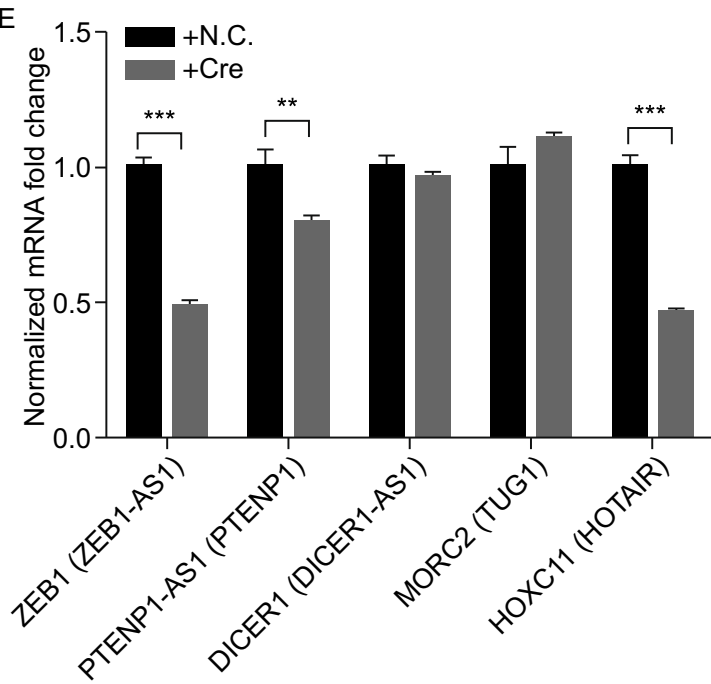

B

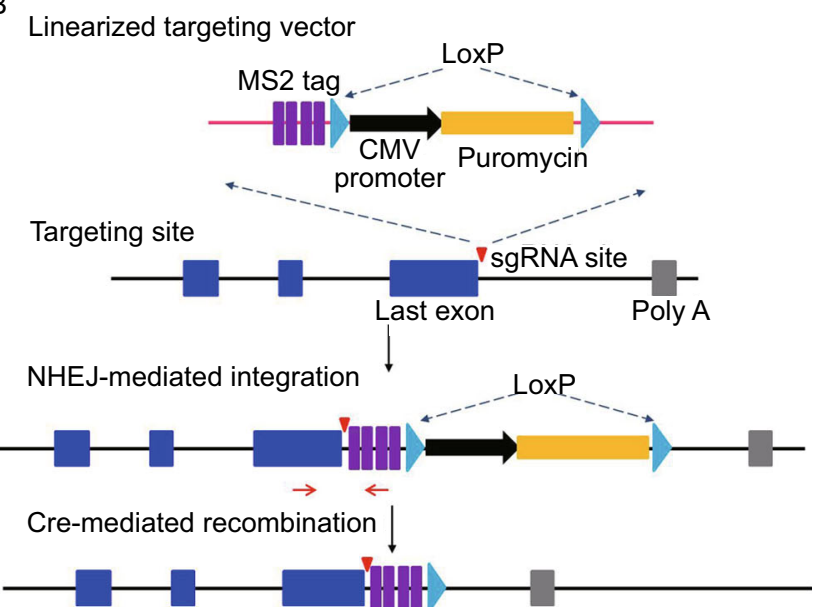

D
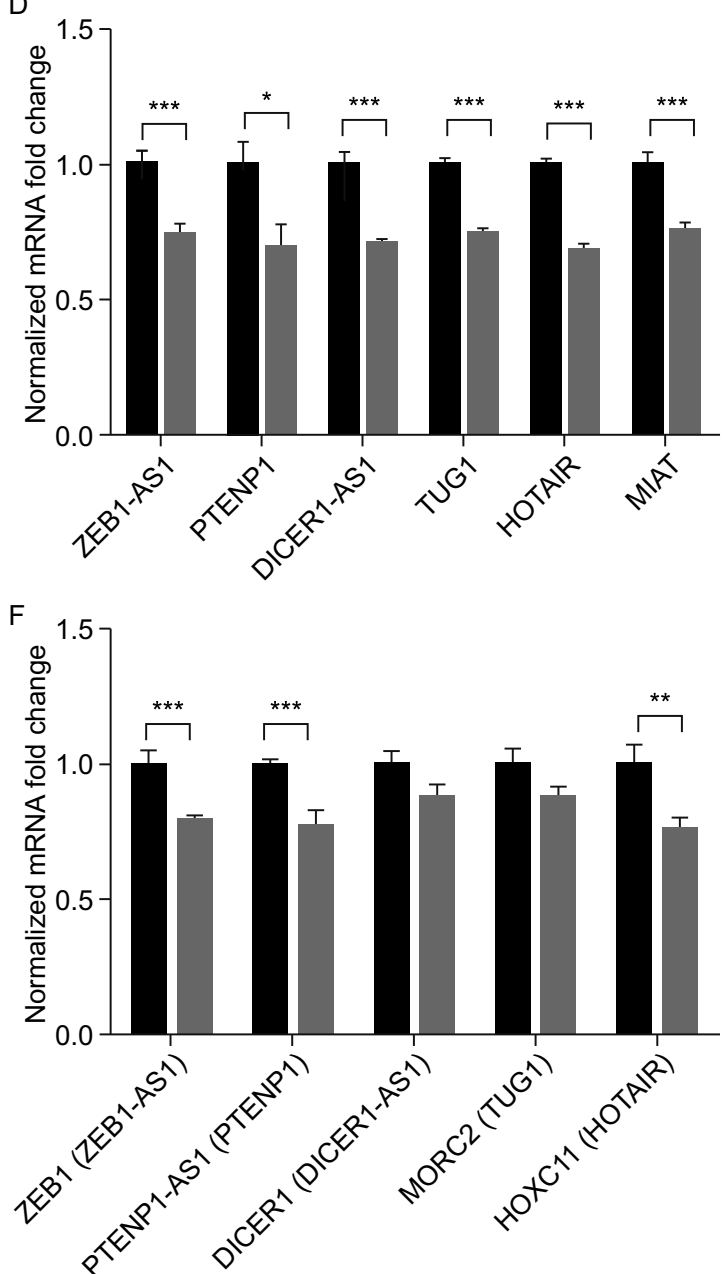

Figure 2. Reversion of targeted insertion near transcriptional termination by Cre-mediated recombination. (A and $B$ ) $A$ scheme for CRISPR/Cas9-mediated targeted insertion, which is similar to Fig. 1A; Cre-mediated reversion of targeted insertion is as follows: targeted insertion could be deleted with Cre recombinase delivery. (C and D) Quantitative PCR analysis of targeted IncRNA expression in 293T cells with established targeted insertion after transfecting with Cre expression vector. (E and F) Quantitative PCR analysis of neighboring genes' expression in 293T cells with established targeted insertion after transfecting with Cre expression vector. GAPDH was used as internal control in $(\mathrm{C})$ and $(\mathrm{F})$ analysis. All experiments were performed independently three times and one representative result was shown. ${ }^{*} P<0.05,{ }^{* \star} P<0.01$, ${ }^{* * *} P<0.005$ (two-sided Student's $t$-test). 
examined in 293T cells. Established targeted insertions were examined and confirmed at the genomic level with PCR for all six IncRNAs similarly as described above (Fig. S4B). Then we evaluated the corresponding IncRNA expressions in 293T cells containing established targeted insertion and found that the expression of ZEB1-AS1, PTENP1, HOTAIR, and MIAT was stimulated while DICER1-AS1 and TUG1 were inhibited (Fig. S4C), which indicated complicated regulatory effects for targeted insertion at transcriptional start sites. Expression status of neighboring genes was also examined and it was revealed that the expression of ZEB1, PTENP1-AS1, and HOXC11 was upregulated (Fig. S4D), in consistent with the regulatory relationship observed in cells with established targeted insertion near transcriptional termination sites. Cre-mediated reversion of targeted insertion was also evaluated in 293T cells containing established targeted insertion at transcriptional start sites and expected expression reversion was observed. Upregulated IncRNAs including ZEB1-AS1, PTENP1, HOTAIR, and MIAT were inhibited while downregulated IncRNAs including DICER1AS1 and TUG1 were induced by Cre recombinase (Fig. S4E). LncRNA-regulated target genes including ZEB1, PTENP1-AS1, and HOXC11 were also reversed by Cre recombinase (Fig. 4F), confirming the regulatory relationship between ZEB1/PTENP1-AS1/HOXC11 and ZEB1-AS1/ PTENP1/HOTAIR, respectively.

CRISPR/Cas9-mediated targeted insertion has been used for the tagging and correction of protein-coding RNAs (Schmid-Burgk et al., 2016; Suzuki et al., 2016). Here we combined CRISPR/Cas9 with modified gene trap vectors for IncRNA tagging and expression manipulation. Together with the Cre-LoxP system, we further established conditional targeted insertion system for IncRNA expression manipulation for the first time, which was valuable for IncRNA functional analysis. Recently several IncRNA manipulation tools have been established and applied to a large number of IncRNAs (Zhu et al., 2016; Liu et al., 2017). However, as described above, limitations still exist, making it difficult to elucidate the functions of IncRNAs overlapping with other genes and stimulate IncRNA expression specifically (Quinn and Chang, 2016), which could be overcome by our CTRL system. Taken together, our system provides a valuable tool for comprehensive analysis of IncRNA functions and might be used for high-throughput screening in the future.

\section{FOOTNOTES}

We thank Yuefang Zhang and Shifang Shan for their help in experiments. We also thank members of the entire Qiu lab for their help and suggestions. Tian-Lin Cheng is supported by The Knowledge Innovation Program of CAS 2014KIP205, Shanghai Sailing Program 15YF1414200, and NSFC \#31600826. Zilong Qiu is supported by NSFC \#91432111, \#31625013.

Tian-Lin Cheng and Zilong Qu declares that they have no conflict of interest. This article does not contain any studies with human or animal subjects performed by any of the authors.

\section{Tian-Lin Cheng $^{\varpi_{(D)}}$, Zilong Qiu ${ }^{凶}$}

Institute of Neuroscience, State Key Laboratory of Neuroscience, CAS Center for Excellence in Brain Science and Intelligence

Technology, Chinese Academy of Sciences, Shanghai 200031, China

$\triangle$ Correspondence: tlcheng@ion.ac.cn (T.-L. Cheng), zqiu@ion.ac.cn (Z. Qiu)

\section{OPEN ACCESS}

This article is distributed under the terms of the Creative Commons Attribution 4.0 International License (http://creativecommons.org/ licenses/by/4.0/), which permits unrestricted use, distribution, and reproduction in any medium, provided you give appropriate credit to the original author(s) and the source, provide a link to the Creative Commons license, and indicate if changes were made.

\section{REFERENCES}

Beaulieu YB, Kleinman CL, Landry-Voyer AM, Majewski J, Bachand F (2012) Polyadenylation-dependent control of long noncoding RNA expression by the poly(A)-binding protein nuclear 1. PLoS Genet 8:e1003078

Carninci P, Kasukawa T, Katayama S, Gough J, Frith MC, Maeda N, Oyama R, Ravasi T, Lenhard B, Wells C et al (2005) The transcriptional landscape of the mammalian genome. Science 309:1559-1563

Chu C, Zhang QC, da Rocha ST, Flynn RA, Bharadwaj M, Calabrese JM, Magnuson T, Heard E, Chang HY (2015) Systematic discovery of Xist RNA binding proteins. Cell 161:404-416

Cong L, Ran FA, Cox D, Lin S, Barretto R, Habib N, Hsu PD, Wu X, Jiang W, Marraffini LA et al (2013) Multiplex genome engineering using CRISPR/Cas systems. Science 339:819-823

Engreitz JM, Sirokman K, McDonel P, Shishkin AA, Surka C, Russell P, Grossman SR, Chow AY, Guttman M, Lander ES (2014) RNARNA interactions enable specific targeting of noncoding RNAs to nascent Pre-mRNAs and chromatin sites. Cell 159:188-199

Guttman M, Garber M, Levin JZ, Donaghey J, Robinson J, Adiconis X, Fan L, Koziol MJ, Gnirke A, Nusbaum C et al (2010) Ab initio reconstruction of cell type-specific transcriptomes in mouse reveals the conserved multi-exonic structure of lincRNAs. Nat Biotechnol 28:503-510

Liu SJ, Horlbeck MA, Cho SW, Birk HS, Malatesta M, He D, Attenello FJ, Villalta JE, Cho MY, Chen Y et al (2017) CRISPRi-based genome-scale identification of functional long noncoding RNA loci in human cells. Science. doi:10.1126/science.aah7111

Mali P, Yang L, Esvelt KM, Aach J, Guell M, DiCarlo JE, Norville JE, Church GM (2013) RNA-guided human genome engineering via Cas9. Science 339:823-826

Quinn JJ, Chang HY (2016) Unique features of long non-coding RNA biogenesis and function. Nat Rev Genet 17:47-62

Schmid-Burgk JL, Honing K, Ebert TS, Hornung V (2016) CRISPaint allows modular base-specific gene tagging using a ligase-4dependent mechanism. Nat Commun 7:12338 
Stanford WL, Cohn JB, Cordes SP (2001) Gene-trap mutagenesis: past, present and beyond. Nat Rev Genet 2:756-768

Suzuki K, Tsunekawa Y, Hernandez-Benitez R, Wu J, Zhu J, Kim EJ, Hatanaka F, Yamamoto M, Araoka T, Li Z et al (2016) In vivo genome editing via CRISPR/Cas9 mediated homology-independent targeted integration. Nature 540:144-149

Ulitsky I, Bartel DP (2013) lincRNAs: genomics, evolution, and mechanisms. Cell 154:26-46
Wapinski O, Chang HY (2011) Long noncoding RNAs and human disease. Trends Cell Biol 21:354-361

Zhu S, Li W, Liu J, Chen CH, Liao Q, Xu P, Xu H, Xiao T, Cao Z, Peng $J$ et al (2016) Genome-scale deletion screening of human long non-coding RNAs using a paired-guide RNA CRISPR-Cas9 library. Nat Biotechnol 34:1279-1286

Electronic supplementary material The online version of this article (doi:10.1007/s13238-017-0464-9) contains supplementary material, which is available to authorized users. 